\title{
Modeling the reduction of gross lithium erosion observed under high-flux deuterium bombardment
}

\author{
T. Abrams ${ }^{\mathrm{a},}{ }^{*}$, M.A. Jaworski ${ }^{\mathrm{a}}$, R. Kaita ${ }^{\mathrm{a}}$, J.H. Nichols ${ }^{\mathrm{a}}$, D.P. Stotler ${ }^{\mathrm{a}}$, G. De Temmerman ${ }^{\mathrm{b}}$, \\ M.A. van den Berg ${ }^{\text {b }}$, H.J. van der Meiden ${ }^{\text {b }, ~ T . W . ~ M o r g a n ~}{ }^{b}$ \\ ${ }^{a}$ Princeton Plasma Physics Laboratory, Princeton, NJ, 08543, USA. \\ ${ }^{b}$ FOM Institute DIFFER-Dutch Institute For Fundamental Energy Research, Trilateral Euregio Cluster, \\ Associate EURATOM-FOM, BL-3430 BE Nieuwegein, The Netherlands.
}

\begin{abstract}
Both thin $(<1 \mu \mathrm{m})$ and thick $(\sim 500 \mu \mathrm{m})$ lithium films under high-flux deuterium and neon plasma bombardment were studied in the linear plasma device Magnum-PSI at ion fluxes $>10^{24}$ $\mathrm{m}^{-2} \mathrm{~s}^{-1}$ and surface temperatures $<700{ }^{\circ} \mathrm{C}$. During Ne plasma exposures, Li erosion rates inferred from measurements of Li-I radiation exceed Langmuir Law evaporation, but no previous results exist to benchmark the binary collision approximation (BCA) and thermal sputtering measurements. Measured Li erosion rates during D plasma bombardment were compared to the adatom-evaporation model of thermal sputtering with an additional reduction term to account for the relative Li/D composition of the Li film. This model captures the qualitative evolution of the Li erosion yield but still overestimates the measured erosion by a factor of 5-10. This suggests that additional refinements to the mixed-material model are needed.
\end{abstract}

PACS: 52.70.Kz, 52.40.Hf, 68.15.+e

PSI-21 Keywords: liquid lithium, sputtering yield, deuterium retention, Magnum-PSI

*Corresponding author address: PPPL, P.O. Box 451, Princeton, NJ 08543-0451

*Corresponding author e-mail: tabrams@pppl.gov

Presenting author: Tyler Abrams

Presenting author e-mail: tabrams@pppl.gov

\section{Introduction}


Liquid lithium is an attractive plasma facing component (PFC) for a DEMO reactor because it improves confinement $[1,2]$ and protects the underlying substrate from high particle fluxes $[3,4]$. However Li evaporation and the strong enhancement of the Li sputter yield at elevated temperatures observed on low-flux experiments $[5,6]$ implies that the maximum Li temperature permitted on such devices may be unacceptably low. Recently thin $(<1 \mu \mathrm{m}) \mathrm{Li}$ films on TZM molybdenum substrates were studied in the Magnum-PSI linear plasma device [7] during ion fluxes $\Gamma_{i} \leq 2 \times 10^{24} \mathrm{~m}^{-2} \mathrm{~s}^{-1}$, electron temperatures $T_{e}<5 \mathrm{eV}$, electron densities $n_{e}>$ $10^{20} \mathrm{~m}^{-3}$, and Li surface temperatures $T_{\mathrm{Li}}<800{ }^{\circ} \mathrm{C}$ [8]. For comparison, the NSTX-U and ITER divertors are expected to experience $\Gamma_{D+}>10^{23} \mathrm{~m}^{-2} \mathrm{~s}^{-1}$ and $10^{24} \mathrm{~m}^{-2} \mathrm{~s}^{-1}$, respectively $[9,10]$. Measured temperature-dependent $\mathrm{Li}$ erosion yields were significantly reduced relative to those observed on a low-flux device [6] and were lower even than the predictions of Langmuir Law evaporation alone.

It was proposed in [8] that the observed discrepancy in erosion rates was due to deuterium retention in the Li layer during Magnum-PSI experiments. In this paper, a model is developed to predict how fast a $\mathrm{Li}$ layer will saturate with $\mathrm{D}$ and what level suppression of $\mathrm{Li}$ erosion is expected as a function of the $\mathrm{D} / \mathrm{Li}$ concentration. To test this model, $\mathrm{D}$ plasma exposures were conducted on thick $(\sim 500 \mu \mathrm{m}) \mathrm{Li}$ films that are expected take 5-10 seconds to approach a 1:1 D/Li concentration ratio under fluxes $>10^{24} \mathrm{~m}^{-2} \mathrm{~s}^{-1}$. In addition, Li erosion behavior was measured via neon plasma bombardment to verify that $\mathrm{Li}$ erosion rates were at least greater than Langmuir Law evaporation for deuterium-free Li coatings. 


\section{Theory}

At elevated temperatures the erosion rate of a Li PFC is enhanced over simple collisional sputtering by evaporation and temperature-dependent (thermal) sputtering [5]. A model for thermal Li sputtering involving the evaporation of surface adatoms has been proposed $[11,12]$. This model predicts the following relation for thermal Li sputtering:

$$
Y_{\text {thermal }}\left(T_{\mathrm{Li}}\right)=\frac{Y_{a d}}{1+A \exp \left(\frac{E_{\text {eff }}}{k_{B} T_{\mathrm{Li}}}\right)}
$$

where $k_{B}$ is Boltzmann's constant and $Y_{a d}$ is a temperature-independent adatom yield. $A$ and $E_{\text {eff }}$ are constants associated with the competing processes of adatom evaporation and recombination. Equation 1 is applied to measured Li thermal sputtering yields [6] using $Y_{a d}, \mathrm{~A}$, and $E_{\mathrm{eff}}$ as fitting parameters. The resulting fit values are $Y_{\mathrm{ad}}=2.9, A=9.6 \times 10^{-6}$, and $E_{\mathrm{eff}}=0.70 \mathrm{eV}$.

Magnum-PSI experiments are needed to measure Li erosion rates at higher temperatures and fluences than PISCES-B could obtain. Li films exposed to high D fluences will retain implanted D in up to a 1:1 ratio [13]. TRIM [14] simulations on a 1:1 Li:D mixture predicts $Y_{\text {coll,LiD }} \approx 0.1 Y_{\text {coll,Li }}$ for $20 \mathrm{eV} \mathrm{D \rightarrow Li} \mathrm{sputtering.} \mathrm{While} \mathrm{the} \mathrm{BCA} \mathrm{assumption} \mathrm{begins} \mathrm{to} \mathrm{break}$ down around $30 \mathrm{eV} \mathrm{[15],} \mathrm{reasonable} \mathrm{agreement} \mathrm{between} \mathrm{theory} \mathrm{and} \mathrm{experiment} \mathrm{at} \mathrm{lower} \mathrm{energies}$ has been found in many cases [16]. Assuming the sputter yield is reduced in proportion to the ratio $\beta$ of $\mathrm{D} / \mathrm{Li}$ atoms on the target surface, we estimate $Y(\beta)=Y(1-\beta)+0.1 Y \beta=Y(1-$ $0.9 \beta$ ). It is assumed that the sputter contribution from reflected ions re-depositing on the surface is negligible. The $\mathrm{Li}$ vapor pressure $p_{\mathrm{Li}}$ of $\mathrm{Li} / \mathrm{D}$ mixtures as a function of $\beta$ has also been wellcharacterized [17]. The predicted Li erosive flux for a mixed-material Li/D layer is written as:

$$
\Gamma_{L i}\left(T_{\mathrm{Li}}, \beta, \Gamma_{D+}\right)=\Gamma_{D+}\left[Y_{\text {coll }}+Y_{\text {thermal }}\left(T_{L i}\right)\right](1-0.9 \beta)+\frac{p\left(T_{L i}, \beta\right)}{\sqrt{2 \pi m_{L i} k_{B} T_{L i}}}
$$


where $m_{L i}$ is the Li atomic mass $(6.941 \mathrm{amu})$ and the third term of Equation 2 represents the Langmuir law formulation [18] of the evaporation rate.

The time-dependent value of $\beta$ on a $\mathrm{Li}$ surface is estimated with 1-D diffusion simulations that incorporate the measured diffusion coefficient $\alpha\left(T_{L i}\right)$ for hydrogen in liquid Li [19]. It is assumed that $\alpha$ and the deuterium recycling coefficient $R$ are reduced by $1-\beta$ and $\alpha=0$ for solid Li. The predicted time evolution of $\beta$ (averaged over the first $50 \mathrm{~nm}$ of the surface) for a typical Magnum-PSI discharge is shown in Figure 1 for several Li thicknesses. The surface remains fully saturated with $\mathrm{D}$ until the Li melting point $\left(180^{\circ} \mathrm{C}\right)$ is reached, when $\beta$ drops to near zero before gradually increasing at a rate governed by the thickness of the Li layer, the D flux, and the Li temperature.

\section{Experimental apparatus and procedure}

The adatom-evaporation mixed-material Li erosion model was tested in the linear plasma device Magnum-PSI [7]. Details of the experimental apparatus and procedure for exposing thinfilm $(<1 \mu \mathrm{m})$ Li samples $25 \mathrm{~mm}$ in diameter to D ion fluxes $\Gamma_{D+} \leq 2 \times 10^{24} \mathrm{~m}^{-2} \mathrm{~s}^{-1}$ at electron densities $n_{e} \leq 8 \times 10^{20} \mathrm{~m}^{-3}$ and temperatures $T_{e} \leq 3 \mathrm{eV}$ are described in [8]. The experimental procedure is similar for the neon plasma exposures described herein. Neon was used instead of helium in order to prevent fuzz formation on the underlying Mo substrate. Copper magnetic coils of peak current $I_{B}$ of $14 \mathrm{kA}$ provide an axial magnetic field of $0.25 \mathrm{~T}$ at the target. A new sample geometry was developed for exposing macroscopically thick $(\leq 500 \mu \mathrm{m}) \mathrm{Li}$ layers to the plasma. $64 \mathrm{~mm}$ diameter TZM molybdenum samples were machined with a $25 \mathrm{~mm}$ diameter, $500 \mu \mathrm{m}$ deep well in the center. A small Li ingot was placed in the well and the TZM Mo sample was heated on a hot plate inside an Ar glove box to a temperature of $\sim 400{ }^{\circ} \mathrm{C}$. After the Li metal liquefied and completely filled the well, any excess was removed with a straight-edge. 
A thin stainless steel (SS) cover was placed over the well and samples were placed in aluminized Mylar heat-seal bags to minimize air contamination.

When mounting the Li-wetted samples on the Magnum-PSI target holder, the SS shim stock cover was removed immediately before sealing up and pumping down the vacuum vessel. The lithium was exposed to atmosphere for about 20-30 seconds during this process. After installation on the target holder the Li samples appeared black in color (Figure 2a), indicating formation of oxide layers on the surface. The Li layers were exposed to several Ar plasma discharges to sputter away impurities from the surface, during which the Li reached a temperature of $\sim 350^{\circ} \mathrm{C}$. After these pulses the surface had transformed to the highly reflective state (Figure 2b) characteristic of pure lithium. In addition, measurements from optical emission spectroscopy (OES) showed little to no evidence of neutral oxygen (O-I) emission $(777 \mathrm{~nm})$ or hydroxide $(\mathrm{OH})$ emission $(308-310 \mathrm{~nm})$ in the plasma by the end of the Ar discharges. This implies that minimal oxygen or hydrogen impurities were present in the Li film immediately prior to D plasma bombardment.

\section{Results}

The procedure for obtaining measurements of the $\mathrm{D}$ ion flux and Li temperature, as well as the analysis methods used to infer Li erosion rates from a Phantom camera with a Li-I (670.8 $\mathrm{nm}$ ) filter, have been previously described [8]. The temperature-dependent Li yields under 20 $\mathrm{eV}$ and $40 \mathrm{eV} \mathrm{Ne}$ ion bombardment are shown in Figure 3, along with the predicted $\mathrm{Li}$ evaporation rate from the Langmuir Law. Li erosion rates are equal to or higher than Langmuir Law evaporation. Since no previous measurements exist for $\mathrm{Ne} \rightarrow \mathrm{Li}$ sputtering, Equation 2 is applied to this data as an empirical fit function. The values of the fitting parameters are $\mathrm{Y}_{\text {coll }}=$ $8.3 \times 10^{-4}, Y_{a d}=0.010, A=8.2 \times 10^{-6}$, and $E_{\text {eff }}=0.81 \mathrm{eV}$ for the $20 \mathrm{eV}$ case and $\mathrm{Y}_{\text {coll }}=0.019, \mathrm{Y}_{\mathrm{ad}}=$ 
$4.2 \times 10^{6}, \mathrm{~A}=57$, and $\mathrm{E}_{\text {eff }}=0.88 \mathrm{eV}$ for the $40 \mathrm{eV}$ case. It should be noted that measurements on low-flux devices give similar thermal sputtering behavior independent of ion energy $[6,11]$, which was not observed here.

During the Ar cleaning process on thick Li layers, significant macroscopic melt motion of the liquid $\mathrm{Li}$ is observed, which alters the Li thickness from its initial value of $500 \mu \mathrm{m}$. Thus $\beta(t)$ was calculated for Li thicknesses ranging from $50-500 \mu \mathrm{m}$ (see Figure 1) to quantify the level of uncertainty introduced in $\beta(t)$. Any mass transport that occurs via melt motion during the D plasma discharge itself not accounted for. The time-dependent $\mathrm{D}$ ion flux $\Gamma_{D+}$ for this discharge is shown in Figure 1. These values were obtained by measuring $n_{e}$ and $T_{e}$ profiles with $1 \mathrm{~Hz}$ frequency via Thomson scattering [20] and linearly interpolating between each time point. Prior to the first measurement point at $t=1 \mathrm{~s}, n_{e}$ and $T_{e}$ are assumed proportional to the magnet current $I_{B}$. After the last measurement point at $t=6 \mathrm{~s}, n_{e}$ and $T_{e}$ are assumed constant.

The measured temperature-dependent erosion yield $\Gamma_{\mathrm{Li}} / \Gamma_{\mathrm{D}+}$ for $20 \mathrm{eV} \mathrm{D}$ bombardment of the Li layer is shown in Figure 4. Measured yields are higher than in [8], which is consistent with calculations that a thick Li coating will saturate with D more slowly, and thus less reduction of the sputter yield via preferential sputtering is expected. The predicted Li yields based on the mixed-material adatom-evaporation model (Equation 2) are overlaid. Also overlaid are the predictions of the pure Li thermal-spike model described in [8]. Since collisional sputtering and evaporation provide negligible contributions to the erosion rate between $300{ }^{\circ} \mathrm{C}$ and $650{ }^{\circ} \mathrm{C}$, this is effectively a plot of the thermal sputtering term only. The error band on the predicted Li yields stems from uncertainty in the $\mathrm{D} / \mathrm{Li}$ concentration $\beta$ at the surface. The error range on $\beta$ by the end of the discharge is $0.45-0.99$. The modeling efforts in Section 2 qualitatively capture the measured temperature dependence of $Y_{L i}$, but an absolute factor of 5-10 discrepancy is 
observed. These results suggest that additional refinements to the modeling of the Li-D mixedmaterial surface are needed; possible refinements are discussed below.

\section{Discussion and Summary}

Previous results showed that a discrepancy existed between the measured Li erosion rates in low-flux experiments on PISCES-B and high-flux exposures on Magnum-PSI, which were even lower than predictions from Langmuir Law evaporation alone. Measured values of $\mathrm{Li}$ erosion during 20 and $40 \mathrm{eV} \mathrm{Ne}$ ion bombardment remain higher than Langmuir Law evaporation, as expected. No data sets exist for $\mathrm{Ne} \rightarrow \mathrm{Li}$ bombardment on low-flux experiments from which to benchmark the measured collisional and thermal sputtering yields on MagnumPSI. But the fact that the measured Li erosion rates are equal to or higher than Langmuir Law evaporation, a well-established lower bound for the erosion rate of pure Li, suggests that there is not some inherent inaccuracy in the measurement technique.

It was hypothesized that the discrepancy between high-flux and low-flux devices was caused by significant D retention in Li layers exposed Magnum-PSI plasmas. Predictions of the rate of $\mathrm{D}$ accumulation in Li coatings were performed using a 1D diffusion model. The expected reduction of the $\mathrm{D} \rightarrow \mathrm{Li}$ sputtering yield (via preferential sputtering) was estimated as a function of the $\mathrm{D} / \mathrm{Li}$ concentration. Measurements of the $\mathrm{D} \rightarrow \mathrm{Li}$ erosion rate on macroscopically thick $\mathrm{Li}$ coatings were performed on Magnum-PSI in the temperature range $200{ }^{\circ} \mathrm{C}<T_{L i}<650{ }^{\circ} \mathrm{C}$. A factor of 5-10 discrepancy was still observed between predictions and measurements of $\mathrm{Li}$ erosion after mixed-material effects were taken into account. This observed discrepancy suggests that the mixed-material model of Li erosion developed in this work may not be completely realistic. A more realistic model of preferential sputtering could be obtained utilizing a simulation code that incorporates dynamic material composition changes, such as TRIDYN 
[21]. In addition, the previous calculations assume that the $\mathrm{D} / \mathrm{Li}$ ratio $\beta$ cannot exceed unity. If a non-equilibrium situation exists such that the surface is temporarily "over-saturated" with D atoms, the effective Li yield would be further reduced, although such an over-saturation has never been observed in surface science experiments. Finally, this model does not include the effects of chemical formation of lithium deuteride (LiD), which could further reduce sputtering since the surface binding energy of $\mathrm{LiD}(2.26 \mathrm{eV})$ is higher than that of pure $\mathrm{Li}(1.67 \mathrm{eV})$. The discrepancy between predictions and measurements of Li erosion under high-flux D bombardment suggest that one or more of these effects must be incorporated to create a realistic model for mixed-material $\mathrm{Li} / \mathrm{D}$ erosion.

\section{Acknowledgements}

This work is supported by US DOE contracts DE-AC02-09CH11466 and the US DOE Fusion Energy Sciences Fellowship. FOM authors are supported by the Stichting voor Fundamenteel Onderzoek der Materie (FOM), which is financially supported by the Nederlandse Organisatie voor Wetenschappelijk Onderzoek (NWO).

\section{References}

[1] H.W. Kugel et al., J. Nucl. Mater. 415 (2011) S400-S404.

[2] G.Z. Zuo et al., J. Nucl. Mater. 415 (2011) S1062-S1066.

[3] T. Abrams et al., J. Nucl. Mater. 438 (2013) S313-S316.

[4] G. Mazzitelli et al., Fusion Eng. Des. 85 (2010) 896-901.

[5] J.P. Allain et al., Phys. Rev. B 76, 205434 (2007).

[6] R.P. Doerner et al., J. Nucl. Mater. 290-293 (2001) 166-172. 
[7] G. De Temmerman et al., Fus. Eng. Des. 88 6-8 (2013) 483-487.

[8] T. Abrams et al., Fus. Eng. Des. (2014), http://dx.doi.org/10.1016/j.fusengdes.2014.04.054.

[9] M.A. Jaworski et al., J. Nucl. Mater. 438 (2013) S384-S387.

[10] P. Norajitra et al., Fus. Eng. Des. 83 (2008) 893-902.

[11] R.P. Doerner et al., J. Appl. Phys. 95 (2004) 4471.

[12] R.P. Doerner et al., J. Nucl. Mater. 337-339 (2005) 877-881.

[13] M.J. Baldwin et al., Fusion Eng. Des. 61-62 (2002) 231-236.

[14] J.F. Ziegler, The Stopping Range of Ions in Matter, Vols. 2-6, Pergamon Press, 1977-1985.

[15] W. Eckstein, Computer Simulations of Ion-Solid Interaction, Berlin: Springer, 1995.

[16] R. Behrisch and W. Eckstein, Sputtering by Particle Bombardment, Berlin: Springer, 2007.

[17] E.E. Shpil'rain et al., Thermophysical properties of lithium hydride, deuteride, and tritide and of their solutions with lithium, New York: American Institute of Physics, 1987.

[18] I. Langmuir, Mon. Weather Rev. 45 (1917) 452.

[19] H. Moriyama et al., J. Nucl. Mater. 191-194 (1992) 190-193.

[20] H.J. van der Meiden et al., Rev. Sci Instrum. 83, 123505 (2012).

[21] W. Möller and W. Eckstein, Nuc. Instr. and Meth., 2 (1984) 814-818. 


\section{Figure Captions}

1. The predicted time evolution of $\beta$ in a typical Magnum-PSI discharge for several different Li thicknesses based on a 1D diffusion model. The inferred ion flux $\Gamma_{D+}$ and the Li temperature $T_{L i}$ are also provided.

2. Images of the Li surface (a) before and (b) after Ar plasma discharge cleaning.

3. Inferred atomic $\mathrm{Li}$ yields vs. $\mathrm{Li}$ temperature for $20 \mathrm{eV}$ and $40 \mathrm{eV}$ neon plasma bombardment of a $1 \mu \mathrm{m} \mathrm{Li} \mathrm{layer.} \mathrm{Fits} \mathrm{to} \mathrm{the} \mathrm{data} \mathrm{using} \mathrm{Equation} 2$ are shown with solid lines. The predicted $\mathrm{Li}$ evaporation rate for the $20 \mathrm{eV}$ case is shown with a dashed line.

4. Inferred atomic Li yields for $20 \mathrm{eV}$ D plasma bombardment of Li. Predictions using the adatom-evaporation mixed-material model as well as the pure Li thermal spike model described in [8] are overlaid (dashed red line). 


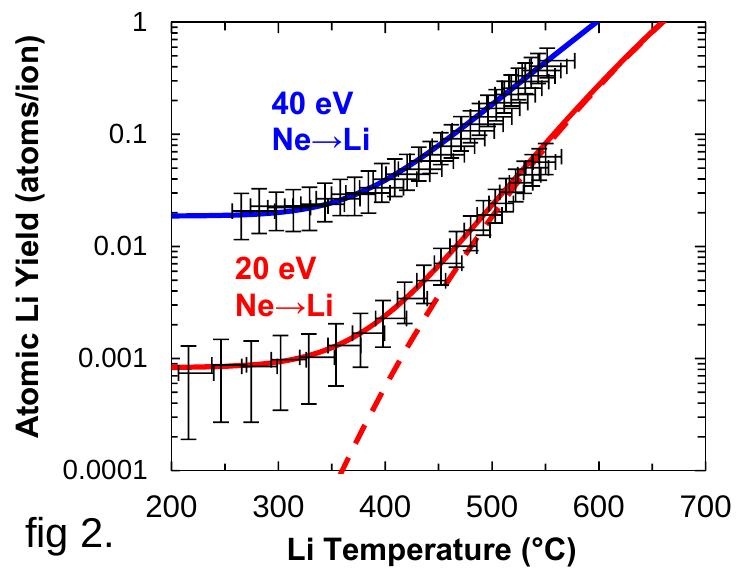




\section{$2.5 \mathrm{~cm}$}

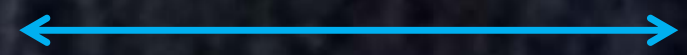

(a) fig 3

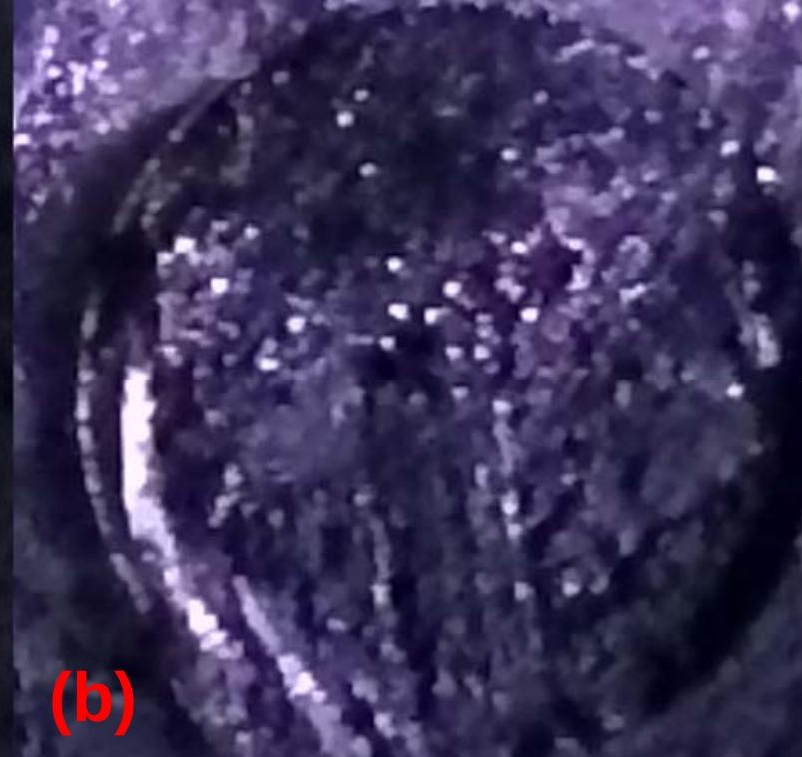




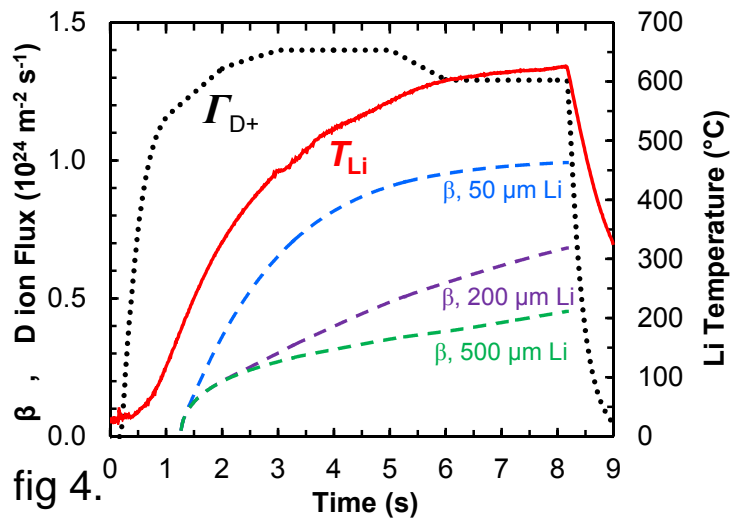

